\title{
REPRESENTACIONES DE LA POBREZA Y LA DESIGUALDAD INFANTIL EN LA CIENCIA DEL DESARROLLO ${ }^{1}$ EN ARGENTINA.
}

\author{
Mariana C. SMULSKI ${ }^{2}$ \\ Cecilia E. HIDALGO ${ }^{3}$ \\ Sebastián J. LIPINA ${ }^{4}$
}

\section{Resumen}

El presente trabajo propone explorar representaciones sobre la pobreza y la desigualdad en distintas investigaciones que abordan la problemática de la pobreza y el desarrollo cognitivo infantil, llevadas a cabo por profesionales en ciencia cognitiva, psicología y educación en Argentina. Para realizar el análisis se seleccionaron publicaciones científicas desde el año 2009 hasta la actualidad. Se buscó poner en diálogo estas investigaciones con producciones antropológicas y sociológicas sobre estos temas, recuperando los aportes a la discusión que pretenden fomentar una relación colaborativa entre las distintas disciplinas científicas.

Palabras clave: POBREZA - DESIGUALDAD - DESARROLLO COGNITIVO INFANTIL- REPRESENTACIONES- INTERDISCIPLINA

\begin{abstract}
This paper aims to explore representations of poverty and inequality in different researchs addressing the problem of poverty and child cognitive development, carried out by professionals in Cognitive Science, Psychology and Education in Argentina. For the analysis, scientific publications were selected since 2009 until today. It was sought
\end{abstract}

\footnotetext{
${ }^{1}$ En la literatura anglosajona contemporánea, las líneas de investigación psicológica o neurocientífica que se ocupan de algún aspecto del desarrollo humano, han comenzado a reunirse bajo el nombre de ciencia del desarrollo (e.g., Bornstein y Lamb, 2010). En Argentina, tal construcción no se ha dado aún, por lo que cabe preguntarse si sería un esfuerzo valioso para la construcción interdisciplinaria de conceptos y metodologías sobre el desarrollo de diferentes atributos (i.e., neurbiológicos, emocionales, cognitivos, conductuales y socio-culturales).

${ }^{2}$ Becaria doctoral UBA. Instituto de Ciencias Antropológicas (FFyL, UBA) - Unidad de Neurobiología Aplicada (UNA, CEMIC-CONICET). mcsmulski@gmail.com

${ }^{3}$ Instituto de Ciencias Antropológicas (FFyL, UBA).

${ }^{4}$ Unidad de Neurobiología Aplicada (UNA, CEMIC-CONICET)
}

Fecha de recepción del artículo: Julio 2015

Fecha de evaluación: Octubre 2015 
to put these in dialogue with anthropological and sociological research productions on these issues, taking into account the contributions to the discussion that aim to foster a collaborative relationship between different scientific disciplines.

Keywords: POVERTY - INEQUALITY - CHILD COGNITIVE DEVELOPMENTREPRESENTATIONS- INTERDISCIPLINARY WORK

\section{Résumé}

Ce travail propose explorer des représentations sur la pauvreté et l'inégalité sur des différentes investigations qui prennent la problématique de la pauvreté et le développement cognitif des enfants experimentés par des professionnels en science cognitive, psycologie et éducation en Argentine. Pour réaliser cet analyse on a selectionné des publications scientifiques à partir de l'année 2009 jusqu' à nos jours. On a cherché mettre en dialogue ces investigations avec des productions anthropologiques et sociologiques sur ces sujets en recupérant les apports à la discussion qui prétendent fomenter une relation collaborative entre les différentes disciplines scientifiques.

Mots clés: PAUVRETÉ - INÉGALITÉ - DÉVELOPPEMENT COGNITIF DES ENFANTS- REPRÉSENTATIONS- INTERDISCIPLINE

\section{Introducción}

Los conceptos y teorías que se forman en el campo científico representan el mundo e intervienen en él, por lo que pueden concebirse como instrumentos que sirven tanto para pensar como para hacer (Hacking, 1983; Hidalgo y Palleres, 2011). Tal afirmación cabe a las representaciones sobre la pobreza y la desigualdad, cuyo proceso de formación conceptual abordaremos en el presente trabajo.

Debido a que la ciencia cognitiva busca actualmente pensar nuestra corporalidad en constante intercambio con el medio -perspectiva en la que las dimensiones biológica y social se encontrarían entrelazadas-, en los últimos años se han incorporado a los análisis del desarrollo cognitivo, argumentos y evidencias propios de las ciencias sociales y humanas (Lipina, 2014; Rose y Abi-Rached, 2013). Las ciencias sociales han destacado asimismo la importancia del cuerpo y lo corporal en sus análisis pero ello no ha redundado en una incorporación de la dimensión biológica, con excepción de algunos abordajes aislados, provenientes principalmente de otros países (Lende y Downey, 2012; Brown y Seligman, 2009; Chiao, 2009; Dominguez et. al. 2009; Leatherman, 2008). En este contexto, se encuentra abierta una oportunidad para explorar las múltiples maneras en que la biografía, la sociedad, la cultura e incluso la historia y la política se "hacen carne", se "meten bajo la piel", fomentando una relación colaborativa entre las distintas disciplinas científicas.

Al menos en Argentina, tales esfuerzos interdisciplinarios parecerían ser aún escasos, motivo por el cual estaríamos careciendo de experiencias documentadas que sirvan de antecedente. Por lo tanto, con la intención de comenzar a generar producciones conjuntas, el presente trabajo propone explorar representaciones sobre la pobreza y la desigualdad en distintas investigaciones que abordan la problemática de la pobreza y el desarrollo cognitivo infantil, llevadas a cabo por profesionales que desarrollan sus actividades en el contexto de las ciencias cognitivas, de la psicología del desarrollo y la educación en Argentina. Para análisis se decidió introducir intencionalmente el concepto de "desigualdad" e hipotetizar sobre los supuestos que le atribuyen las investigaciones seleccionadas de la ciencia del desarrollo infantil, dado que en la mayoría de las mismas no se observa un uso sistemático de un concepto que sí se ha 
vuelto central en las investigaciones contemporáneas de las ciencias sociales y humanas, desplazando en gran medida al de pobreza. En forma específica, se buscará poner en diálogo estas investigaciones con los aportes de las producciones antropológicas y sociológicas respecto de estos temas, buscando recuperar la contribución de los distintos autores a la discusión. En este sentido, se valorizan los esfuerzos llevados a cabo desde distintos campos científicos por contribuir con la problemática, debido a que la reducción de las desigualdades continúa siendo un desafío pendiente que persiste en todo el mundo y en particular en nuestra región (Piketty y Saez, 2014; Ravallion, 2014). A su vez, deben resaltarse las dificultades que se presentan al momento de intentar elaborar estudios adecuados que generen información y herramientas de intervención apropiadas, a ser articuladas con políticas públicas eficaces.

Es amplia la literatura en las ciencias sociales y humanas respecto de las limitaciones que presenta el concepto de pobreza y los distintos indicadores construidos a lo largo del tiempo para identificarla y medirla. Si bien a partir de la crisis del modelo neoliberal, en América Latina y en nuestro país se privilegiaron los estudios de la pobreza de manera unidimensional, en los últimos años se han desarrollado métodos multidimensionales incluyendo otras variables más allá del ingreso, que ha caracterizado a los primeros (Kessler, 2014). En este sentido, cabe aclarar que la propuesta de elaborar indicadores multidimensionales es sostenida por organismos multilaterales como el Banco Mundial, o la Organización de las Naciones Unidas (ONU) y sus distintos programas y comisiones, como el Programa de Naciones Unidas para el Desarrollo (PNUD) o la Comisión Económica para América Latina y el Caribe (CEPAL) (Sipicker et. al., 2009).

Tomaremos como ejemplo un documento elaborado por el PNUD en el año 2010, en donde se define el Índice de Pobreza Multidimensional (IPM). En el mismo, se manifiesta la preocupación de reflejar en relación a la pobreza tres dimensiones principales que se encontrarían superpuestas a la dimensión del ingreso: salud, educación y niveles de vida; y dentro de estas tres, distintos indicadores: nutrición, mortalidad infantil, años de instrucción, matriculación escolar, combustible para cocinar, saneamiento, agua, electricidad, piso y bienes. Contemplar estas dimensiones e indicadores permitiría, según el PNUD, la elaboración de un panorama multifacético sobre la pobreza amplio y orientador a la política pública.

No obstante, las definiciones operacionales ${ }^{5}$ del concepto de pobreza propuestas por dichos organismos han seguido despertando críticas, debido a la dificultad que presentan a la hora de integrar y articular las dimensiones de análisis entre sí. Algunos autores sostienen que pensar en términos de multidimensionalidad requiere establecer y contemplar relaciones entre las distintas variables de análisis (Kessler, 2014). Se critica en propuestas de medición como las de los organismos multilaterales, una reunión de indicadores unidimensionales que no modifican en forma significativa la conceptualización de pobreza, sino que tan solo agrupan en conjunto distintas variables que venían siendo contempladas individualmente ${ }^{6}$. Por lo tanto, si bien desde los organismos internacionales se reconoce que la superación de la pobreza no se alcanza

\footnotetext{
${ }^{5}$ Por definiciones operacionales, se comprenden aquellas definiciones que establecen determinadas variables -llamadas indicadores- para identificar empíricamente las propiedades de dichas variables que pueden medirse u observarse (Samaja, 2010).

${ }^{6} \mathrm{Si}$ bien esta problemática excede los objetivos del presente trabajo, es importante mencionarla y proponer su discusión con mayor profundidad en futuros trabajos.
} 
solo con el crecimiento económico ${ }^{7}$, las herramientas de medición y las políticas públicas consiguientes no acompañan tal reconocimiento. Una propuesta multidimensional debería poder no solo reunir distintos indicadores bajo una misma rúbrica, sino articular e interrelacionar las distintitas variables de análisis y contemplar las dinámicas que producen situaciones de pobreza y desigualdad.

En los últimos años, al menos en las ciencias sociales y humanas, se han privilegiado los estudios de la desigualdad. Esta categoría, se presenta desde estos campos de conocimiento como superadora de la pobreza, en tanto esta última tiende a identificar grupos más que procesos y relaciones. Por ello se propone recuperar las concepciones de los diversos autores que contribuyen a pensar a la pobreza como un corolario de la desigualdad, en tanto que esta noción permite “(...) reinscribir a la pobreza dentro de la dinámica social y entenderla como un subproducto de las inequidades" (Kessler, 2014: 17). La definición propuesta por Tilly permite identificar a la desigualdad humana en tanto

“(...) distribución despareja de atributos entre un conjunto de unidades sociales tales como los individuos, las categorias, los grupos o las regiones" (2000: 38). El autor busca así recuperar la multiplicidad de dimensiones de la experiencia -no sólo la económica- en que la desigualdad puede desarrollarse, así como sus respectivas dinámicas de cambio.

Este hecho no ha implicado descartar el concepto de pobreza, sino que en pos de construir una mirada multidimensional se resalta la necesidad de articularlo dentro de procesos más amplios y contemplar las dinámicas y temporalidades propias en que las distintas desigualdades sociales se encuentran, yuxtaponen y refuerzan (Reygadas, 2008). Desde esta mirada, algunos autores han propuesto el concepto de interseccionalidad destacando la inconveniencia de analizar dimensiones aisladas de la desigualdad (como por ejemplo, clase, género y etnia), en tanto que las mismas se configuran en una articulación compleja (Jenlin, 2015).

Veremos en el presente trabajo cómo algunas investigaciones en el área del desarrollo infantil asumen el desafío de la multidimensionalidad y cómo en su proceso las respuestas a este desafío pueden ser aún profundizadas, a partir de la sinergia que emerge de tomar en cuenta enfoques en clave interdisciplinaria. Debe resaltarse que la intención de este análisis es incentivar el diálogo entre distintas perspectivas teóricas, a partir de la propuesta de explorar cómo distintos campos de conocimiento construyen una representación de la pobreza o la desigualdad y una intervención diferencial sobre ella. Ello implica poner el énfasis en distintas dimensiones de análisis que podrían ser sin embargo compatibles entre sí y no excluyentes.

\section{Antecedentes}

Los estudios acerca de los efectos de la pobreza sobre el desempeño cognitivo y el académico fueron iniciados en los contextos disciplinarios de la psicología del desarrollo y la educación. En forma sintética, los resultados de los estudios sobre efectos realizados durante la segunda mitad del siglo XX, han encontrado una disminución de los coeficientes de desarrollo motor y mental durante los primeros treinta y seis meses de vida; así como también en los coeficientes verbales y de

\footnotetext{
${ }^{7}$ Ver página web del PNUD: http://www.latinamerica.undp.org/content/rblac/es/home/idh-regional/hdr$\underline{2015-2016 /}$
} 
ejecución de pruebas generales de inteligencia en niños de edad preescolar y escolar. A ello se agrega menor cantidad de años de educación completados, una mayor incidencia de dificultades de aprendizaje y tasas más altas de ausentismo y abandono escolar (Bradley y Corwyn, 2002; Yoshikawa et.al., 2012). Respecto al lenguaje, los estudios actuales en el contexto de estas disciplinas también verifican perfiles de desempeño modulados por el nivel socioeconómico en indicadores de vocabulario, habla espontánea, desarrollo de la gramática y estilos y habilidades de comunicación (Hoff, 2003). Durante la última década estos abordajes continuaron desarrollándose con la incorporación de factores que combinan información de diferentes contextos de desarrollo y de métodos de análisis más complejos, que en conjunto han contribuido con mejorar la comprensión de los mecanismos de mediación involucrados en la modulación de la pobreza. Por ejemplo, Najman y colegas (2009) analizaron cómo los cambios en la pobreza familiar entre el embarazo, la infancia temprana y la adolescencia predicen los cambios en el desarrollo cognitivo en la adolescencia. Sus resultados confirmaron tendencias previas que sugerían que la pobreza experimentada en cualquiera de esas etapas se asociaba a una reducción del desempeño cognitivo, y que la exposición a pobreza por períodos extensos era más perjudicial que la experimentada sólo durante un período (NICHD \& Human Development Early Child Care Research Network, 2005). En todos los casos, la definición de pobreza se asoció a alguna variante del método de ingresos o bien a los niveles educativos y ocupaciones logrados por los padres.

Asimismo, durante la última década y media han comenzado a realizarse los primeros estudios neurocientíficos que evalúan la asociación entre diferentes formas de pobreza y su impacto en los procesamientos cognitivos y de lenguaje básicos. Tales estudios han asociado diferentes formas de deprivación socioeconómica, también basadas por lo general en criterios de ingreso, educación y ocupación parentales- con diferentes aspectos del procesamiento durante la ejecución de tareas cognitivas y de lenguaje, con y sin evaluación concomitante de la activación de diferentes redes neurales. Por ejemplo, respecto a las influencias de la pobreza en el procesamiento del lenguaje a nivel neural, Noble y colegas (Nayar, 2006), estudiaron a niños de entre 6 y 9 años que habían sido seleccionados por tener puntajes promedio bajos en tareas de conciencia fonológica, y encontraron que diferentes niveles de ingreso se asociaban a relaciones específicas entre el nivel de procesamiento fonológico y la activación neural en áreas posteriores del hemisferio izquierdo. Asimismo, en un estudio reciente Raizada y colegas (2008), encontraron una relación directa entre el nivel de ingreso y educativo de los padres de niños de 5 años y el grado de activación de áreas frontales asociadas al habla. Además del lenguaje, otro de los sistemas neurocognitivos más explorados durante la última década, es el de los procesos cognitivos de control asociados a la activación de redes que involucran diferentes áreas prefrontales. En estudios con poblaciones de niños de diferentes edades, el nivel de ingreso y la insatisfacción de necesidades básicas ha sido asociado a una reducción en el desempeño en tareas con demandas de atención, control inhibitorio y memoria de trabajo desde el primer año de vida en adelante (ej., Hackman y Farah, 2009 ; Lipina, 2014 ; Lipina y Colombo, 2009), así como también con diferencias en los patrones de activación evaluados con electroencefalografía (ej., D'Angiulli y otros, 2008 ; Kishiyama y otros, 2009 ; Stevens y otros, 2009)

Las investigaciones antropológicas en relación al desarrollo infantil comenzaron a publicarse alrededor del año 1920, a partir de la producción de registros coloniales que 
buscaban documentar la variedad de condiciones en que los niños eran criados alrededor del mundo, y las maneras en que estas prácticas diferían de las occidentales. Desde sus comienzos, este análisis transcultural desafió la manera en que se concebía a la infancia, dado que se evidenciaban distintas condiciones y resultados en la crianza que al momento no podían ser anticipados por los expertos europeos y norteamericanos (LeVine y New, 2008). En este contexto se destacan los trabajos de Franz Boas, Margaret Mead, Bronislaw Malinowski, Meyer Fortes y Ruth Benedict (LeVine y New, 2008). El mayor aporte de la antropología en los últimos años consistió en documentar, describir y analizar formas alternativas de crianza, desde la niñez hasta la adolescencia, que ponen en cuestionamiento los estándares universales alrededor de lo que se considera el camino "normal" del desarrollo a nivel general, desde la perspectiva occidental. Desde la consideración del aspecto cultural, estos estándares han sido analizados teniendo en cuenta su carga moral y los presupuestos biológicos en los que se apoyan. Por otra parte, desde la consideración de los aspectos biológicos muchos antropólogos - al igual que los psicólogos del desarrollo durante todo el siglo XX- han señalado en relación al desarrollo infantil, que la potencialidad de cada niño cambia y debe ser estudiada en relación a su ambiente particular, sus características comportamentales, genéticas, culturales, simbólicas y nutricionales (LeVine y New, 2008). Si bien la antropología ha abordado problemáticas en relación a la infancia y los diversos contextos socioeconómicos y culturales en los que la misma se desarrolla, la problemática del desarrollo cognitivo infantil en contextos de pobreza con tal especificidad ha sido escasamente abordada. En este sentido, se identifica actualmente el trabajo de Lende (2012) quien analiza el modelo de investigación elaborado por neurocientíficos en Estados Unidos para estudiar el impacto del estatus socioeconómico en el desarrollo cerebral, al que llama "poverty poisons the brain model" (2012; 184). En dicho trabajo el autor resalta la potencialidad de estas investigaciones para estudiar conjuntamente e interdisciplinariamente las maneras en que la cultura y la sociedad dan forma al desarrollo neurocognitivo.

Desde la antropología y la sociología a su vez, se ha abordado por un lado la formación de conceptos como la pobreza, la exclusión social o la desigualdad y la manera en que estos permiten concebir la realidad social (Hidalgo y Palleres, 2011). Por otro lado, se observan investigaciones que se centran en la descripción y análisis de las distintas configuraciones socioculturales y socioeconómicas, aumentando en los últimos años los estudios sobre la desigualdad (ej., Jenlin, 2015; Perez Sáinz, 2014; Faur, 2014; Kessler, 2013; Grimson y Baeza, 2011). A modo de ejemplo, puede citarse el análisis desarrollado por Grimson y Baeza (2011) quienes en la ciudad de Comodoro Rivadavia (provincia de Chubut, Argentina), constatan un desacople entre la estructura de ingresos y el prestigio, siendo los trabajadores petroleros el grupo de mayores ingresos y menor jerarquía simbólica, así como el de mayor estigmatización. En este sentido, presentan argumentos a favor de pensar que la redistribución económica no sería una condición suficiente para la reducción multidimensional de la desigualdad (Grimson y Baeza, 2011). Esta concepción puede identificarse a su vez en las argumentaciones de distintos investigadores que colaboraron en el año 2009 en la construcción de un glosario internacional sobre pobreza (Spicker et. al., 2009).

A su vez, Wilkinson y Pickett (2009) proveen argumentos a favor de privilegiar el concepto de desigualdad frente al de pobreza material. Según estos autores, la visión de que los problemas sociales son provocados por la carencia material, implicaría que las sociedades "más ricas" tendrían menos problemas que las otras sociedades. Sin 
embargo, este hecho no sucede dado que muchos países que alcanzan un gran crecimiento económico, no presentan un aumento equivalente en términos de grado de satisfacción, salud, bienestar o felicidad de las personas (ver Figura 1). En efecto, cuestiones sociales como la salud mental, el desempeño académico, el abuso de drogas o el embarazo adolescente, no se correlacionan con la falta de recursos, sino más bien con las diferencias que se presentan al interior de cada sociedad.

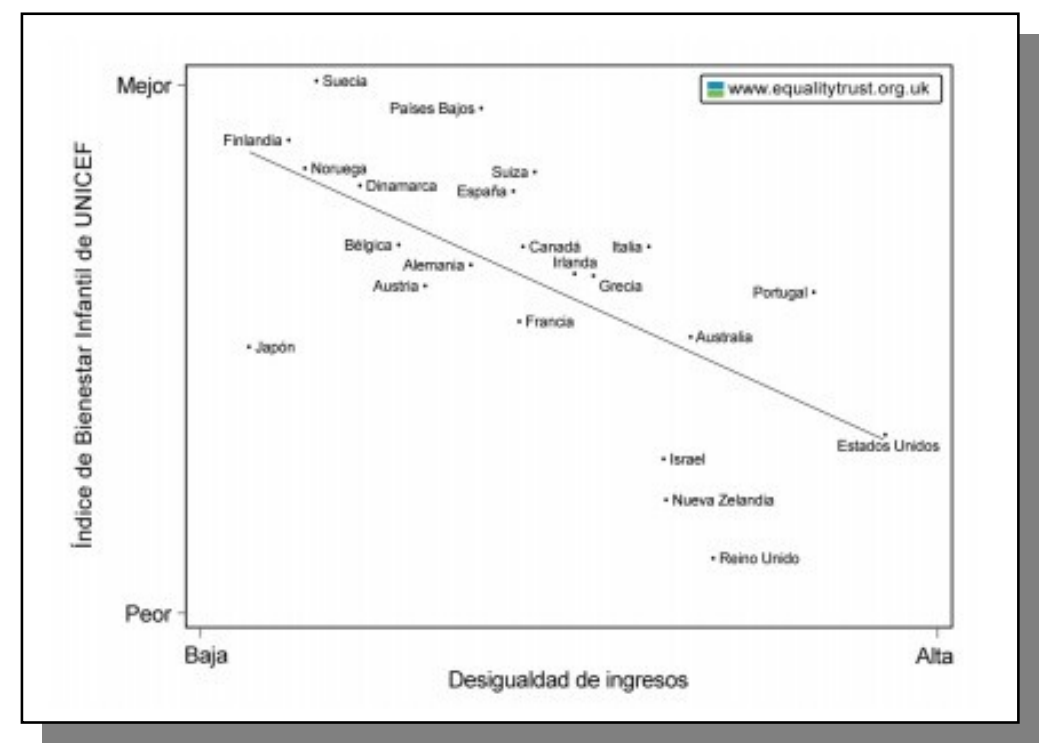

Figura 1. Índice de UNICEF del bienestar infantil en países ricos en relación a la desigualdad de renta ${ }^{8}$.

\section{Metodología}

Para realizar el análisis, se seleccionaron aquellas investigaciones científicas de la ciencia del desarrollo que abordaron la relación entre la pobreza y el desarrollo cognitivo infantil en Argentina desde el año 2009 hasta la actualidad, con el fin de considerar los últimos cinco años de producción. Con las palabras clave "pobreza", "desarrollo cognitivo" y "Argentina" se consultaron las bases de Google académico, Scielo, Redalyc y CONICET ${ }^{9}$ - en este último caso, para identificar grupos de investigación con producción en el área.

Dicha búsqueda dio como resultado la identificación de tres grupos de investigación, que tienen producciones contemporáneas sobre el tema: (i) CIIPME- CONICET (Centro Interdisciplinario de Investigaciones en Psicología Matemática y Experimental); (ii) Facultad de Psicología, Universidad Nacional de Tucumán, Argentina; (iii) UNA, CEMIC-CONICET (Unidad de Neurobiología Aplicada). Para el análisis se seleccionaron los siguientes trabajos: del grupo (i) investigaciones $\mathrm{A}, \mathrm{B}, \mathrm{C}^{10}$; del grupo

\footnotetext{
${ }^{8}$ Pobreza en la infancia, perspectivas. Agosto de 2010. Políticas y estrategias. Disponible en la página web de UNICEF: http://www.unicef.org/spanish/socialpolicy/files/Insights_August2010_SPA.pdf ${ }^{9}$ CONICET: www.conicet.gov.ar

${ }^{10}$ Se identifican las investigaciones con letras, para facilitar su comparabilidad en el texto del trabajo. En el listado de referencias se explicitan a qué trabajos corresponden cada una de las letras.
} 
(ii) investigaciones D y E; del grupo (iii) investigación F. Dichos grupos se componen de profesionales que desde distintas disciplinas teorizan acerca de la manera en que la pobreza produce efectos sobre el desarrollo cognitivo en la infancia, en tanto período particularmente sensible a los efectos del ambiente, y realizan propuestas sobre posibles maneras de intervenir en la problemática.

A partir de la lectura de las publicaciones científicas del período y de los grupos seleccionados, se tomaron como ejes para el análisis los siguientes aspectos: (1) el criterio de definición de pobreza utilizado; (2) la metodología utilizada para la obtención de datos sobre pobreza; (3) la utilización y sentido de uso del término "desigualdad"; (4) la construcción de un abordaje directo o mediado de la relación entre pobreza y el desarrollo cognitivo infantil; y (5) las propuestas de intervención de las investigaciones consideradas.

En relación al punto (1), teniendo en cuenta lo abordado en introducción y antecedentes, se buscó identificar el criterio tomado por los investigadores para definir y medir pobreza en la población a analizar (ya sean criterios unidimensionales $\mathrm{o}$ multidimensionales) y los distintos conceptos afines que hacen referencia a condiciones socioeconómicas de la población. A continuación en el punto (2), se identificaron las distintas metodologías que los investigadores utilizaron para recolectar datos sobre pobreza y en relación a ello el criterio utilizado para seleccionar los lugares en donde se iba a desarrollar la investigación. En el punto (3) se buscó identificar si en las investigaciones seleccionadas era utilizado el concepto de desigualdad y de qué manera era utilizado. De no aparecer explícitamente el término, se procedió a interpretar a posteriori el posible sentido otorgado por los investigadores, teniendo en cuenta la construcción de las argumentaciones y la utilización de terminologías afines. En relación al punto (4) debe aclararse que por abordaje directo o mediado de la pobreza se comprende: (a) aquellas investigaciones que plantean una influencia directa del contexto socioeconómico sobre el desarrollo cognitivo; y (b) aquellas que proponen la existencia de uno o muchos mecanismos que mediatizan la experiencia y deben considerarse con especificidad en el análisis. Por ejemplo, en la literatura occidental suelen incluirse a los siguientes mediadores del impacto de la pobreza sobre el desarrollo cognitivo: la salud física y la nutrición peri y pos natal, las condiciones ambientales del hogar y la estimulación, las interacciones entre padres e hijos, la salud mental de los padres, los estilos de crianza y los recursos materiales y sociales del barrio (Lipina et. al. 2013). Finalmente, en el punto (5) se consideraron las propuestas de intervención sobre la problemática y los ámbitos en donde debería desarrollarse la misma.

Siguiendo los ejes de análisis citados, se procedió a construir una matriz de datos que se completó con la lectura y análisis de las publicaciones científicas.

\section{Resultados}

A continuación se presenta la matriz de datos confeccionada siguiendo los criterios especificados en la sección de metodología. En términos generales la matriz permite identificar las coincidencias y las disidencias principales entre las investigaciones seleccionadas. Particularmente, todas ellas coinciden en la elaboración de un modelo causal en donde los aspectos socioeconómicos (contemplados desde distintos criterios y métodos de medición) producen un efecto en el desarrollo de determinadas funciones

cognitivas. Dicho modelo causal, varía en las investigaciones $\mathrm{C}$ y $\mathrm{F}$ en tanto que el estatus socioeconómico, no es visto como un mecanismo próximo que puede interactuar 
de manera directa con el desarrollo cognitivo infantil, sino que interactúa a través de factores locales, identificados como mediadores. Específicamente en la investigación $\mathrm{C}$ se propone la identificación de un único mediador -el nivel de instrucción materno (NIM)- mientras que en la investigación $F$ se contemplan múltiples variables mediadoras que serán desarrolladas en la siguiente sección.

Por otro lado, cabe aclarar que cada investigación seleccionada se centra en distintos aspectos del desarrollo cognitivo y del lenguaje infantil: la investigación A se centra en el aprendizaje de letras; la investigación $\mathrm{B}$ en las habilidades lectoras; en la investigación $\mathrm{C}$ se analizó el desempeño en tareas de lenguaje, atención y memoria; en la investigación D se estudiaron los procesamientos cognitivos simultáneo y secuencial; en la investigación E se analizaron las habilidades sociales facilitadoras o inhibidoras de la socialización; y en la investigación $\mathrm{F}$ se analizó el control atencional, la memoria de trabajo y la planificación, para comparar el desempeño en el control ejecutivo no verbal. 
TABLA 1. Representaciones de pobreza y desigualdad.

\begin{tabular}{|c|c|c|c|c|c|c|}
\hline & $\begin{array}{c}\text { INVESTIGACIÓN } \\
A\end{array}$ & $\begin{array}{c}\text { INVESTIGACIÓN } \\
B\end{array}$ & $\begin{array}{c}\text { INVESTIGACIÓN } \\
C\end{array}$ & $\begin{array}{c}\text { INVESTIGACIÓN } \\
D\end{array}$ & $\begin{array}{c}\text { INVESTIGACIÓN } \\
E\end{array}$ & $\begin{array}{c}\text { INVESTIGACIÓN } \\
F\end{array}$ \\
\hline 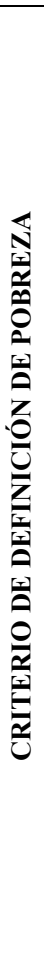 & $\begin{array}{l}\text { Se menciona } \\
\text { "nivel } \\
\text { socioeconómico } \\
\text { bajo" y "contexto } \\
\text { de pobreza". Se } \\
\text { define "nivel } \\
\text { socioeconómico" } \\
\text { partir del nivel } \\
\text { educativo y } \\
\text { ocupacional de los } \\
\text { adultos } \\
\text { responsables. }\end{array}$ & $\begin{array}{l}\text { Se menciona } \\
\text { "contexto de } \\
\text { pobreza", "nivel } \\
\text { socioeconómico } \\
\text { bajo", "Barrios } \\
\text { carenciados", } \\
\text { "poblaciones } \\
\text { marginales". Solo } \\
\text { se define "nivel } \\
\text { socioeconómico" } \\
\text { en base a la } \\
\text { ocupación del } \\
\text { adulto responsable } \\
\text { de los niños, } \\
\text { ocupación que fue } \\
\text { categorizada a } \\
\text { partir de la "escala } \\
\text { de Sautú". }\end{array}$ & $\begin{array}{l}\text { Se menciona } \\
\text { "estrato } \\
\text { socioeconómico } \\
\text { bajo". También: } \\
\text { "coeficiente } \\
\text { socioeconómico": } \\
\text { obtenido en base a } \\
\text { los ingresos de la } \\
\text { familia. Para } \\
\text { analizar las } \\
\text { diferencias entre } \\
\text { los grupos } \\
\text { seleccionados se } \\
\text { tomaron en cuenta } \\
\text { cuatro indicadores } \\
\text { socioeconómicos } \\
\text { del "Método } \\
\text { Social Graffar- } \\
\text { Mendez", que } \\
\text { evalúa cuatro } \\
\text { variables: } \\
\text { profesión del jefe } \\
\text { de familia, nivel } \\
\text { de instrucción de } \\
\text { la madre, } \\
\text { principal fuente de } \\
\text { ingreso de la } \\
\text { familia y } \\
\text { condiciones de } \\
\text { alojamiento. }\end{array}$ & $\begin{array}{l}\text { Se menciona } \\
\text { "desigualdad } \\
\text { social", } \\
\text { "pobreza", } \\
\text { "niveles de } \\
\text { pobreza", } \\
\text { "contexto } \\
\text { socioeconómico", } \\
\text { "contextos } \\
\text { socioculturales } \\
\text { desfavorecidos", } \\
\text { "vulnerabilidad } \\
\text { social". Se toma } \\
\text { como criterio: } \\
\text { cuadro de } \\
\text { desnutrición. } \\
\text { También se utiliza } \\
\text { un indicador NES, } \\
\text { tomando las } \\
\text { variables de } \\
\text { educación y } \\
\text { ocupación de los } \\
\text { padres. }\end{array}$ & $\begin{array}{l}\text { El "NES bajo" se } \\
\text { determinó por la } \\
\text { combinación del } \\
\text { nivel de educación } \\
\text { y ocupación del } \\
\text { jefe de hogar y } \\
\text { acceso a bienes de } \\
\text { consumo (Cámara } \\
\text { de Empresas de } \\
\text { Investigación } \\
\text { Social y de } \\
\text { Mercado y } \\
\text { Asociación } \\
\text { Argentina de } \\
\text { Marketing, 2006). }\end{array}$ & $\begin{array}{l}\text { Se menciona } \\
\text { "background } \\
\text { socioeconómico", } \\
\text { "estatus } \\
\text { socioeconómico } \\
\text { (SES)". Se } \\
\text { propone revisar el } \\
\text { criterio de } \\
\text { necesidades } \\
\text { básicas e ingreso, } \\
\text { debido a que la } \\
\text { pobreza infantil } \\
\text { involucra aspectos } \\
\text { distintos a la } \\
\text { pobreza en } \\
\text { general. Para } \\
\text { obtener datos } \\
\text { socioeconómicos } \\
\text { se estableció una } \\
\text { escala para } \\
\text { completar (NES). } \\
\text { Se contemplaron } \\
\text { indicadores de } \\
\text { NBI, así como } \\
\text { otros indicadores } \\
\text { asociados con la } \\
\text { experiencia } \\
\text { específica de la } \\
\text { pobreza para los } \\
\text { niños. }\end{array}$ \\
\hline 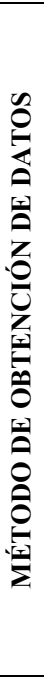 & $\begin{array}{l}\text { Se preseleccionó } \\
\text { una institución } \\
\text { educativa que ha } \\
\text { optado por la } \\
\text { atención de los } \\
\text { niños más pobres. } \\
\text { Luego se obtuvo } \\
\text { información sobre } \\
\text { el nivel educativo } \\
\text { y ocupacional de } \\
\text { los adultos } \\
\text { responsables (no } \\
\text { se aclara cómo). }\end{array}$ & $\begin{array}{l}\text { Se preseleccionó } \\
\text { una institución } \\
\text { educativa y luego } \\
\text { se estableció el } \\
\text { nivel } \\
\text { socioeconómico } \\
\text { de la muestra en } \\
\text { base a la } \\
\text { ocupación del } \\
\text { adulto responsable } \\
\text { de los niños (no se } \\
\text { aclara cómo). }\end{array}$ & $\begin{array}{l}\text { Se tuvieron en } \\
\text { cuenta las } \\
\text { características del } \\
\text { establecimiento } \\
\text { educativo y el } \\
\text { barrio de } \\
\text { pertenencia. El } \\
\text { coeficiente } \\
\text { socioeconómico } \\
\text { de la escuela, } \\
\text { obtenido en base a } \\
\text { los ingresos de la } \\
\text { familia, era } \\
\text { deficitario según } \\
\text { comunicación del } \\
\text { Departamento de } \\
\text { Educación de la } \\
\text { localidad de Santa } \\
\text { Fé. }\end{array}$ & $\begin{array}{l}\text { Para determinar } \\
\text { los niveles de } \\
\text { nutrición y } \\
\text { obtener una } \\
\text { muestra se } \\
\text { utilizaron los } \\
\text { datos de la } \\
\text { Encuesta Nacional } \\
\text { de Nutrición y } \\
\text { Salud, realizada } \\
\text { por el Ministerio } \\
\text { de Salud y } \\
\text { Ambiente de } \\
\text { Argentina en el } \\
\text { periodo 2004- } \\
\text { 2005. Luego para } \\
\text { los datos del NES, } \\
\text { se realizó una } \\
\text { encuesta socio } \\
\text { demográfica. }\end{array}$ & $\begin{array}{l}\text { Se realizó una } \\
\text { encuesta socio } \\
\text { demográfica para } \\
\text { identificar el nivel } \\
\text { socioeconómico. }\end{array}$ & $\begin{array}{l}\text { Se administró a } \\
\text { las madres de los } \\
\text { niños una escala } \\
\text { socioeconómica. } \\
\text { Además se tomó } \\
\text { un cuestionario de } \\
\text { comportamiento } \\
\text { de los niños y se } \\
\text { administró la } \\
\text { escala de } \\
\text { Hamilton de } \\
\text { ansiedad y } \\
\text { depresión para } \\
\text { considerar } \\
\text { aspectos de la } \\
\text { salud mental de la } \\
\text { madre. Luego las } \\
\text { evaluaciones de } \\
\text { los niños fueron } \\
\text { individuales en } \\
\text { escuelas. }\end{array}$ \\
\hline 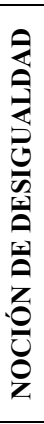 & $\begin{array}{l}\text { No se menciona la } \\
\text { palabra } \\
\text { desigualdad. Se } \\
\text { plantea que el } \\
\text { nivel } \\
\text { socioeconómico } \\
\text { produce } \\
\text { diferencias } \\
\text { individuales en las } \\
\text { habilidades } \\
\text { asociadas al } \\
\text { aprendizaje de } \\
\text { letras. }\end{array}$ & $\begin{array}{l}\text { La pobreza } \\
\text { provoca } \\
\text { desigualdad en las } \\
\text { habilidades } \\
\text { lectoras. }\end{array}$ & $\begin{array}{l}\text { Las desigualdades } \\
\text { en el estatus } \\
\text { socioeconómico } \\
\text { afectan el } \\
\text { desarrollo } \\
\text { cognitivo infantil. }\end{array}$ & $\begin{array}{l}\text { Desigualdad } \\
\text { social en tanto } \\
\text { indicador visible } \\
\text { de pobreza en } \\
\text { América Latina. }\end{array}$ & $\begin{array}{l}\text { No se menciona la } \\
\text { palabra } \\
\text { desigualdad. Se } \\
\text { aborda el impacto } \\
\text { de la pobreza en el } \\
\text { capital simbólico y } \\
\text { cultural. }\end{array}$ & $\begin{array}{l}\text { Desigualdad } \\
\text { social. } \\
\text { Desigualdades } \\
\text { específicas de la } \\
\text { pobreza infantil. }\end{array}$ \\
\hline
\end{tabular}




\begin{tabular}{|c|c|c|c|c|c|c|}
\hline 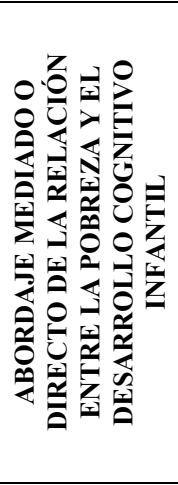 & Directo & Directo & $\begin{array}{l}\text { Mediado. } \\
\text { Principal } \\
\text { mediador: nivel de } \\
\text { instrucción de la } \\
\text { madre (NIM). }\end{array}$ & $\begin{array}{l}\text { Directo (eje en la } \\
\text { desnutrición). Si } \\
\text { bien es un } \\
\text { abordaje directo } \\
\text { de la pobreza } \\
\text { (criterio } \\
\text { nutricional) } \\
\text { presenta } \\
\text { evidencias a favor } \\
\text { de considerar } \\
\text { otros aspectos } \\
\text { coexistentes a los } \\
\text { que llama } \\
\text { "factores de la } \\
\text { pobreza". }\end{array}$ & $\begin{array}{l}\text { Directo. Se } \\
\text { mencionan } \\
\text { variables } \\
\text { socioeconómicas a } \\
\text { tener en cuenta en } \\
\text { futuras } \\
\text { indagaciones, pero } \\
\text { los datos } \\
\text { socioeconómicos } \\
\text { no se cruzan con } \\
\text { los resultados. }\end{array}$ & $\begin{array}{l}\text { Mediado. Presenta } \\
\text { evidencias a favor } \\
\text { de que distintos } \\
\text { mediadores } \\
\text { producen efectos } \\
\text { distintos en el } \\
\text { desarrollo } \\
\text { neurocognitivo. }\end{array}$ \\
\hline 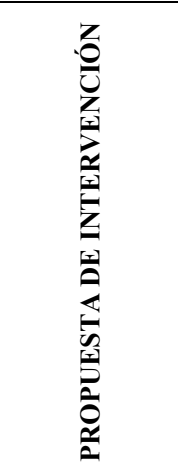 & $\begin{array}{l}\text { Intervención } \\
\text { curricular en la } \\
\text { enseñanza formal. }\end{array}$ & $\begin{array}{l}\text { Generación de } \\
\text { mejores } \\
\text { condiciones } \\
\text { educativas y } \\
\text { estrategias de } \\
\text { acompañamiento } \\
\text { escolar. }\end{array}$ & $\begin{array}{l}\text { Intervención en } \\
\text { ámbitos escolares } \\
\text { y familiares. }\end{array}$ & - & $\begin{array}{l}\text { Intervención } \\
\text { terapéutica. }\end{array}$ & $\begin{array}{l}\text { Se deben analizar } \\
\text { los efectos } \\
\text { variables de la } \\
\text { pobreza en } \\
\text { dimensiones } \\
\text { variables del } \\
\text { desarrollo, en } \\
\text { distintas etapas } \\
\text { del mismo, para } \\
\text { diseñar acciones } \\
\text { que den } \\
\text { oportunidades a } \\
\text { niños que viven } \\
\text { en la pobreza. }\end{array}$ \\
\hline
\end{tabular}

Nota. A: Diuk, B. y Ferroni, M. (2014); B: Diuk, B. y Ferroni, M. (2011); C: Aran-Filippetti, V. y Minzi, M.C. R. DE (2012); D: Lacunza, A. B. (2010); E: Contini, N. et. al. (2010); F: Lipina, S. J. et. al. (2013)

\section{4. a. Definiciones de pobreza}

Siguiendo los datos de la matriz extraídos para el análisis, en cinco de las seis investigaciones seleccionadas orientadas a la infancia (ver Tabla 1), pobreza es definida teniendo en cuenta principalmente el nivel ocupacional y educativo de los padres. A pesar de esta coincidencia, la metodología utilizada para la obtención de datos sobre pobreza, varía en todas las investigaciones y en algunas investigaciones no está explicitada.

Este criterio presenta coincidencias con la definición operacional utilizada en Argentina por el INDEC (Instituto Nacional de Estadística y Censos) para medir lo que se conoce como pobreza estructural. El método de NBI (necesidades básicas insatisfechas) se compone a partir de determinados indicadores de privación, que se utilizan para identificar necesidades poblacionales (Hidalgo y Palleres, 2011). El mismo toma en cuenta principalmente: las condiciones de la vivienda (hacinamiento, tipo de vivienda, condiciones sanitarias), la asistencia escolar de los niños, la capacidad de subsistencia (nivel de educación y ocupación de los adultos) y los servicios comunitarios (acceso al agua potable, red cloacal).

Las investigaciones A, B, C y D comparten con el método NBI -si bien no hacen referencia explícita al mismo- la preocupación por las condiciones educativas y laborales, si bien algunas además combinan dichas variables con otros indicadores. En la investigación F particularmente se pone en cuestionamiento el método NBI, dado que se problematiza la dificultad de medir específicamente la pobreza infantil proponiendo contemplar otros indicadores a fin de reflejar la experiencia de la pobreza en la infancia. 
Con ello se proponen captar mecanismos y procesos que afectan de diversas formas, las distintas etapas del desarrollo cognitivo. Se hace visible pues una preocupación por complejizar los modos de abordar la problemática y construir indicadores más adecuados a la experiencia etaria.

\section{4. b. Representaciones de desigualdad}

Teniendo en cuenta el concepto de desigualdad introducido en secciones previas del presente artículo, resulta pertinente extender las primeras dos preguntas que guían la indagación de Pérez Sáinz (2014) al análisis de la construcción de la relación entre la pobreza y el desarrollo cognitivo en Argentina: ¿desigualdad de qué? y ¿desigualdad entre quiénes?. Como se advirtió anteriormente, dicho concepto no aparece de manera explícita en todas las investigaciones, e incluso en dos de las seleccionadas (investigaciones A y E) no se incluye la palabra, aun cuando se podría inferir una alusión a una o varias situaciones en que los investigadores observan desigualdades generadas a partir de la pobreza, representadas en palabras tales como "diferencias" o "habilidades".

\section{¿Desigualdad de qué?}

Como fue mencionado previamente, cinco de las investigaciones (A, B, C, D y E) coinciden en aplicar un criterio socioeconómico para identificar a las poblaciones que serán objeto de análisis, tomando como indicadores de desigualdad principalmente el nivel educativo y ocupacional de los adultos responsables (ver tabla 1). En la investigación $\mathrm{C}$ los mismos se superponen a su vez con indicadores de ingreso y condiciones de alojamiento. A su vez en la investigación E, se incorpora la posesión de determinados bienes de consumo (si bien no se define el tipo de bienes, se aclara que se siguió el criterio de consumo de la "Cámara de Empresas de Investigación Social y de Mercado" y de la "Asociación Argentina de Marketing"). En este sentido se utilizan términos como "contexto de pobreza", "nivel socioeconómico bajo", "contexto económico y social bajo", todos los cuales aluden a una estratificación espacial pensada en términos de condiciones estructurales deficientes, además de ingresos ordenados en la escala bajo, medio y alto.

Al respecto, Pérez Sáinz (2014) señala que cuando la desigualdad se centra en el ingreso familiar, es decir en la esfera secundaria o de redistribución, se pueden confundir distintas desigualdades bajo una forma monetaria común, cuando estas en realidad podrían corresponder a distintos campos, con distintas dinámicas específicas de poder (ej., laborales y no laborales). Esto cobra particular relevancia a la hora de pensar políticas que busquen reducir inequidades, pues la mirada se enfocaría en procesos en lugar de resultados, en la esfera de la distribución en lugar de la redistribución, en los grupos más que en los individuos (Pérez Sáinz, 2014; 42).

Por otro lado, la investigación $\mathrm{F}$ enmarcada en los enfoques multidimensionales, cuestiona la definición de pobreza a partir del criterio de ingreso; y propone la incorporación de un criterio socio-económico, a partir del establecimiento de una escala multidimensional (NES - nivel económico y social). En este enfoque de investigación, se plantea la dificultad que las medidas y definiciones actuales de pobreza presentan a la hora de reflejar la diversidad de dimensiones fundamentales en el desarrollo infantil, y las necesidades específicas de la niñez que no coinciden necesariamente con las necesidades y experiencias adultas (Lipina et. al., 2011). En este sentido, la desigualdad 
se piensa no sólo en términos económicos y sociales, sino también en términos generacionales (adulto-niño) y en la especificidad de cada etapa del desarrollo en función a cómo las carencias materiales y simbólicas contribuyen a generar diferentes resultados de inequidad, con posibilidades diferentes de transformación por intervención. Esta concepción lleva a pensar en los múltiples pares de categorías en las que se desarrolla la desigualdad (ej., mujer-varón, ciudadano-extranjero) y que pueden incorporarse en futuros análisis (Tilly, 2000).

Además de la desigualdad de ingreso, las investigaciones abordan los correlatos cognitivos de la pobreza, otorgando evidencias a favor de que la deprivación económica genera procesos cognitivos desiguales. En este sentido, cada investigación indaga diferentes aspectos del desarrollo cognitivo infantil: aprendizaje de letras; lectura; vocabulario; comprensión lectora; habilidades sociales; y funciones ejecutivas (ej., atención, control inhibitorio, memoria de trabajo, planificación). Tales evaluaciones se realizan a partir de la administración de pruebas en su mayoría en las escuelas a las que acuden los niños, contribuyendo a un conocimiento detallado sobre los efectos que determinadas condiciones de vida producen sobre el lenguaje y la cognición.

Sin haber entrado específicamente en diálogo debido a la falta de intercambios interdisciplinarios, tal enfoque converge sin proponérselo con las tesis de Tilly (2000), quien nos invita a reflexionar sobre las dificultades que pueden presentar algunas de las propuestas de investigación, al mostrar en ciertos casos resultados colectivos como efectos individuales, y plantea la importancia de no omitir

“(...) los mecanismos causales mediante los cuales la ubicación de la comunidad, la posición del hogar, los recursos parentales y el género producen rendimientos educacionales o las otras características adultas pertinentes" $(2000 ; 36)$ El autor nos advierte que al correlacionar el desempeño de los niños con las posiciones sociales de sus padres, se podría tender a atribuir al contexto familiar las diferencias en el rendimiento, muchas veces sin considerar adecuadamente que funcionarios y docentes dan forma a esos desempeños "(...) mediante sus propias respuestas categoriales a las posiciones sociales de los padres." (2000; 43). Esta preocupación es abordada por la investigación $\mathrm{F}$, que partiendo de una visión ecológica e interrelacional del individuo, trabaja actualmente en la especificación de mecanismos mediadores que permitan elaborar propuestas de intervención superadores de esta dificultad (Lipina, 2013). Estos últimos aspectos considerados nos llevan a la próxima pregunta.

\section{¿Desigualdad entre quiénes?}

Particularmente, en las investigaciones que proponen abordajes directos de la relación entre la pobreza y el desarrollo cognitivo infantil, las desigualdades se establecen a nivel de grupos de niños. En esos casos se suelen identificar grupos a partir del nivel socioeconómico y luego de las pruebas realizadas se procede a analizar y comparar los distintos rendimientos cognitivos.

Particularmente, en las publicaciones científicas de las investigaciones A y B no aparecen datos específicos sobre el proceso de selección de las instituciones educativas a las que se va a acudir, que den cuenta de las características socioeconómicas del lugar. Este aspecto pondría en riesgo tanto la replicabilidad como la construcción del problema de investigación, en tanto que podrían estar actuando en el proceso de selección preconcepciones de sentido común, en relación a la localización de la institución y la población que acude. En dichas investigaciones, la dificultad en el aprendizaje de letras se explicita como una característica del sujeto (una mayor o menor "habilidad") que se 
presenta excluida de un contexto particular; en tanto que se menciona "contexto de pobreza" pero no se problematiza en qué sentido el mismo estaría actuando, o cuáles son los procesos restrictivos o posibilitadores que en el mismo se generan. Este hecho contribuye a una mirada homogeneizadora de la pobreza, dado que se tiende a considerar una relativa uniformidad en las características y experiencias compartidas por sujetos a-situados. Debemos pensar entonces, qué tipo de subjetividad se presupone desde la investigación científica.

Por otro lado, se encuentran las investigaciones que plantean la preponderancia de un mecanismo mediador de la pobreza sobre el desarrollo cognitivo infantil. Las mismas se centran principalmente en la importancia del nivel de instrucción materno (NIM), para predecir el desempeño de niños en tareas cognitivas. Particularmente en la investigación C, se identificaron dos grupos, uno de "estrato socioeconómico bajo" y otro "medio", para analizar el efecto socioeconómico sobre el desempeño en tareas de lenguaje, atención y memoria. A partir de las pruebas realizadas los autores llegan a la conclusión que de las variables socioeconómicas analizadas, el nivel de instrucción de la madre es el principal predictor del desempeño cognitivo de los niños. Por lo tanto, en este contexto la desigualdad se construye entre los niveles de instrucción de la madre y los resultados diferenciales que este hecho produce en los niños.

$\mathrm{Al}$ respecto, resultaría enriquecedor incorporar al análisis los estudios de género sobre la organización social y política del cuidado infantil, en pos de introducir la dimensión histórica y procesual para pensar cómo el mismo se fue considerando una actividad predominantemente femenina y maternal, y la manera en que se ha ido construyendo un "andamiaje simbólico" que coopera con una distribución desigual de poder entre hombres y mujeres (Faur, 2014). La propuesta inicial, podría consistir en promover la contextualización de las categorías, como la de NIM, para que las mismas no se den por sentado como categorías per se y así promover el análisis sobre el desarrollo histórico del rol materno asociado a la educación en el hogar o el cuidado. Si tomamos como ejemplo los documentos de concientización que elabora el Ministerio de Salud Argentino, los mismos están dirigidos a las madres y las imágenes que acompañan los materiales de difusión, son principalmente imágenes de mujeres realizando tareas de cuidado $^{11}$. Teniendo en cuenta estos aspectos, se podría promover dese la investigación científica una elaboración más compleja de la manera en que los aspectos socioculturales se interrelacionan con el desarrollo biológico, y en este caso cognitivo. Se podría pensar a su vez, teniendo en cuenta las propuestas multidimensionales, la posibilidad de integrar el NIM con otros mediadores o insertarlo en una trama más compleja de indicadores interrelacionados.

Por último, en los abordajes que identifican distintos mecanismos mediadores la respuesta a ¿Desigualdad entre quiénes? se vuelve más compleja, debido a que si bien inicialmente se identifica un grupo general a investigar a partir de un criterio económico, se van introduciendo múltiples variables de análisis que configuran un entramado de características psicológicas, sociales, culturales y ambientales, configurando una visión ecosistémica del individuo. Por lo tanto, las desigualdades se conciben a partir de la construcción analítica y la selección de determinados mecanismos mediadores considerados de importancia fundamental en el desarrollo cognitivo infantil, que pueden verse alterados en situaciones de deprivación material y simbólica. Los principales considerados son: la salud física y la nutrición peri y pos

\footnotetext{
${ }^{11}$ http://www.msal.gob.ar/index.php/programas-y-planes
} 
natal, las condiciones ambientales del hogar, la estimulación al aprendizaje en el hogar, las interacciones entre padres e hijos, la salud mental de los padres, los estilos de crianza, los recursos materiales y sociales del barrio, el acceso a la información y a la tecnología, el acceso a la educación y sus recursos.

\section{Conclusión y perspectivas futuras}

En este trabajo, se buscó incorporar el concepto de desigualdad para invitar a pensar cómo la pobreza se inserta en dinámicas y procesos más amplios, que no siempre se encuentran contemplados de manera explícita en las investigaciones de la ciencia del desarrollo infantil. Sin embargo, este hecho no reduce el enorme valor de los aportes que las distintas investigaciones producen al conocimiento del desarrollo cognitivo en la infancia y la preocupación legítima por contribuir a los estudios de la pobreza; sino que manifiesta la importancia de promover espacios de intercambio disciplinarios para contribuir mutuamente a una visión compleja y conjunta de la problemática.

Por otro lado, más allá de las distintas maneras de abordar la problemática de la pobreza y el desarrollo cognitivo infantil y las distintas propuestas de intervención, todas las investigaciones citadas en el presente trabajo presentan evidencias a favor de considerar los efectos tempranos del ambiente sobre el desarrollo. Este punto se vuelve de gran importancia a la hora de pensar la articulación entre la agenda científica y la política pública: la inversión en los periodos más tempranos de la infancia -en lugar de la inversión en programas para revertir efectos en periodos tardíos -podría ayudar a mitigar efectos a largo plazo de la pobreza sobre el desarrollo cognitivo (Hackman y Farah, 2009).

Este señalamiento nos interpela como investigadores. Desde la antropología en relación a la ciencia cognitiva, no ha habido en nuestro país propuestas concretas o programas de investigación que superen la crítica teórica y se inserten en estudios que promuevan la construcción interdisciplinaria. Queda el desafío propuesto a futuro, en pos de promover estudios etnográficos que contribuyan a la elaboración de categorías de análisis que permitan reflejar la complejidad social. En este sentido, Lende (2012), propone cuatro ejes centrales a ser retomados por aquellos investigadores interesados en estudiar los vínculos entre la desigualdad, el desarrollo, la infancia y la salud, en lo que llama un abordaje "neuroantropológico". En primer lugar, propone la utilización de las herramientas conceptuales que proveen los modelos de desarrollo en donde se interrelacionan la causalidad social, el desarrollo biológico, la neuroplasticidad y la epigenética, para comprender los efectos que producen las desigualdades sociales. En segundo lugar, esta temática de investigación permite retomar una pregunta central para la antropología, que es cómo la cultura y la estructura social, dan forma al individuo, en este caso en particular, en relación a los procesos de enculturación y el impacto de la desigualdad. En tercer lugar, las investigaciones que abordan el impacto de la pobreza sobre el cerebro, producen un fuerte modelo retórico que puede ser utilizado por los antropólogos para concientizar sobre los efectos que producen las desigualdades sociales sistémicas y argumentar respecto de las acciones de política pública que deben realizarse para prevenirlos. Por último, teniendo en cuenta los tres puntos anteriores, basándose en las evidencias de la ciencia cognitiva, la antropología puede trabajar de manera aplicada colaborando en el diseño de intervenciones apropiadas a los distintos modos de vida alrededor del mundo. 
A su vez, la antropología puede contribuir a fortalecer el modelo causal que pone en relación el estatus socioeconómico con el desarrollo cognitivo infantil, promoviendo estudios que se centren en la manera en que las condiciones socioeconómicas determinan la mayor o menor disponibilidad de bienes materiales o simbólicos (como por ejemplo, el dominio del lenguaje, el acceso a libros, computadoras, internet, viajes) y de qué manera son producidas y vivenciadas dichas desigualdades. De esta manera, se promueve la elaboración de estrategias basadas en evidencias. La propuesta consiste en establecer un diálogo que integre la riqueza del enfoque etnográfico a partir de reconsiderar a la pobreza y a los diversos indicadores y mediadores utilizados para identificarla, no como atributos de los sujetos, sino como relaciones que sólo pueden ser comprendidas a partir de la experiencia que producen (Rockwell 2009; Cerletti y Gessaghi, 2012; D'Angiulli, Lipina, Olesinska, 2012).

\section{BIBLIOGRAFÍA}

ARÁN-FILIPPETTI, V. y MINZI, M. C. R. DE. (2012): “A Structural Analysis of Executive Functions and Socioeconomic Status in School-Age Children: Cognitive Factors as Effect Mediators"- The Journal of Genetic Psychology- N 173(4)- págs. 393-416.

BORNSTEIN, M. y LAMB, M. (Eds.). (2010). Developmental Science: An Advanced Textbook, Sixth Edition - Psychology Press- New York.

BRADLEY, R. H. y CORWYN, R. F. (2002): "Socioeconomic status and child development.”- Annual Review of Psychology- N53- págs. 371-399.

BROWN, R. A. y SELIGMAN, R. (2009): “Anthropology and cultural neuroscience: creating productive intersections in parallel fields"- Progress in Brain Research- N178págs. 31-42.

CERLETTI, L. B. y GESSAGHI, V. (2013): "Clases sociales, trabajo de campo y desigualdad. Discusiones a partir del enfoque etnográfico.”- PUBLICAR En Antropología y Ciencias Sociales- N 0(13).

CHIAO, J. Y. (2009): “Cultural neuroscience: a once and future discipline."- Progress in Brain Research- N178- págs. 287-304.

CONTINI, N., CORONEL, C. P., LEVIN, M., y HORMIGO, K. (2012): "Las habilidades sociales en contextos de pobreza. Un estudio preliminar con adolescentes de la Provincia de Tucumán.”- Perspectivas en Psicología- N 7(1)- págs. 112-120.

D’ANGIULLI, A., HERDMAN, A., STAPELLS, D., y HERTZMAN, C. (2008): "Children's event-related potentials of auditory selective attention vary with their socioeconomic status." -Neuropsychology-N 22(3)- págs. 293-300.

D'ANGIULLI, A., LIPINA, S. J., y OLESINSKA, A. (2012): "Explicit and implicit issues in the developmental cognitive neuroscience of social inequality."- Frontiers in Human Neuroscience- N6-págs. 1-17.

DIUK, B., y FERRONI, M. (2011): “Aquisição leitora em crianças que crescem em contextos de pobreza: perfis cognitivos de crianças com alto e baixo nível de leitura." Cadernos de Psicopedagogia- N 7(13)- págs. 70-89.

DOMÍNGUEZ D, J. F., LEWIS, E. D., TURNER, R., y EGAN, G. F. (2009): “The brain in culture and culture in the brain: a review of core issues in neuroanthropology." Progress in Brain Research -N178-págs. 43-64.

FAUR, E. (2014): El cuidado infantil en el siglo XXI: mujeres malabaristas en una sociedad desigual - Siglo XXI Editores- Buenos Aires. 
FERRONI, M., y DIUK, B. (2014): “Aprendizaje de letras en niños preescolares de nivel socioeconómico bajo.”- Interdisciplinaria. Revista de Psicología y Ciencias Afines.- N 31(1) - págs.25-37.

GRIMSON, A., y BAEZA, B. (2011): "Desacoples entre nivel de ingreso y jerarquías simbólicas en Comodoro Rivadavia." - Mana.

HACKING, I. (1996): Representar e intervenir- Paidós- México.

HACKMAN, D. A. y FARAH, M. J. (2009): "Socioeconomic status and the developing brain.”-Trends in Cognitive Sciences- N 13(2)- págs. 65-73.

HERMIDA, M. J., SEGRETIN, M. S., LIPINA, S. J., BENARÓS, S. y COLOMBO, J. A. (2010): "Abordajes neurocognitivos en el estudio de la pobreza infantil: consideraciones conceptuales y metodológicas." - International Journal of Psychology and Psychological Therapy- N 10(2)- págs. 205-225.

HIDALGO, C., y PALLERES, G. (2011): "De la pobreza a la exclusión: dinamismo de la formación de conceptos sociales."- Debate público. Reflexión de trabajo social.- N2págs. 73-80.

HOFF, E. (2003): “The Specificity of Environmental Influence: Socioeconomic Status Affects Early Vocabulary Development Via Maternal Speech."- Child Development- N 74(5)- págs. 1368-1378.

JENLIN, E. (2015): "Desigualdades de clase, género y etnicidad/raza: realidades históricas, aproximaciones analíticas.”- Ensambles- N 1- págs. 11-36.

KESSLER, G. (2014): Controversias sobre la desigualdad. Argentina 2003-2013- Fondo de cultura económica- Buenos Aires.

KISHIYAMA, M. M., BOYCE, W. T., JIMENEZ, A. M., PERRY, L. M., y KNIGHT, R. T. (2009): "Socioeconomic disparities affect prefrontal function in children."- Journal of Cognitive Neuroscience- N 21(6)- págs. 1106-1115.

LACUNZA, A. B. (2013): "Procesamiento cognitivo y déficit nutricional de niños en contextos de pobreza."- Psicología y Salud- N 20(1)- págs. 77-88.

LEATHERMAN, T. (2005): "A Space of Vulnerability in Poverty and Health: PoliticalEcology and Biocultural Analysis." - Ethos- N 33(1)- págs. 46-70.

LENDE, D. H. (2012): "Poverty Poisons the Brain." - Annals of Anthropological Practice- N 36(1)- págs. 183-201.

LENDE, D. H., y DOWNEY, G. (EDS.). (2012): The Encultured Brain: An Introduction to Neuroanthropology (1 edition)- The MIT Press- Cambridge.

LEVINE, R. A., y NEW, R. S. (EDS.). (2008): Anthropology and Child Development: A Cross-Cultural Reader (1 edition)- Wiley-Blackwell- Malden, MA.

LIPINA, S. J. (2014): "Biological and Sociocultural Determinants of Neurocognitive Development: Central Aspects of the Current Scientific Agenda" - En Bread and Brain, Education and Poverty - Pontifical Academy of Sciences- págs.1-30- Vatican City.

LIPINA, S. J., y COLOMBO, J. A. (2009): Poverty and Brain Development During

Childhood: An Approach from Cognitive Psychology and Neuroscience.- American

Psychological Association- Washington, DC

LIPINA, S. J., y POSNER, M. I. (2012): "The impact of poverty on the development of brain networks."- Frontiers in Human Neuroscience-N 6 (238)- págs. 1-12.

LIPINA, S. J., SIMONDS, J., y SEGRETIN, M. S. (2011): "Recognizing the child in child poverty."-Vulnerable Children and Youth Studies- N 6(1)- págs. 8-17.

LIPINA, S., SEGRETIN, S., HERMIDA, J., PRATS, L., FRACCHIA, C., CAMELO, J. L., y COLOMBO, J. (2013): "Linking childhood poverty and cognition: environmental 
mediators of non-verbal executive control in an Argentine sample." - Developmental Science- N 16(5)- págs. 697-707.

NAJMAN, J. M., HAYATBAKHSH, M. R., HERON, M. A., BOR, W., O'CALLAGHAN, M. J., y WILLIAMS, G. M. (2009): "The Impact of Episodic and Chronic Poverty on Child Cognitive Development."- The Journal of Pediatrics- N 154(2)- págs. 284-289.

NATIONAL INSTITUTE OF CHILD HEALTH AND HUMAN DEVELOPMENT EARLY CHILD CARE RESEARCH NETWORK. (2005): "Duration and Developmental Timing of Poverty and Children's Cognitive and Social Development From Birth Through Third Grade.” - Child Development- N 76(4)- págs. 795-810.

NAYAR, U. S. (2012): Child and Adolescent Mental Health- SAGE Publications -India.

PÉREZ SÁINZ, J. P. (2014): Mercados y bárbaros. La persistencia de las desigualdades de excedente en América Latina.-FLACSO- Costa Rica.

PIKETTY, T., y SAEZ, E. (2014): “Inequality in the long run.”- Science- N 344(6186)- págs. 838-843- New York, N.Y.

RAIZADA, R. D. S., RICHARDS, T. L., MELTZOFF, A., y KUHL, P. K. (2008):

"Socioeconomic status predicts hemispheric specialisation of the left inferior frontal gyrus in young children."- NeuroImage- N 40(3)-págs. 1392-1401.

RAVALLION, M. (2014): "Income inequality in the developing world."- Science- N 344(6186)- págs. 851-855- New York, N.Y.

REYGADAS, L. (2008): La apropiación: destejiendo las redes de la desigualdad.Anthropos- Barcelona.

ROCKWELL, E. (2009): La experiencia etnográfica: historia y cultura en los procesos educativos- Paidós - Argentina.

ROSE, N., y ABI-RACHED, J. M. (2013): Neuro: The New Brain Sciences and the Management of the Mind- Princeton University Press.

SAMAJA, J. (2010): Epstemología y metodología. Elementos para una teoría de la investigación científica- Eudeba- Buenos Aires.

SPICKER, P., ALVAREZ LEGUIZAMÓN, S., y GORDON, D. (EDS.) (2009): Pobreza, un glosario internacional. CLACSO- Buenos Aires.

STEVENS, C., LAUINGER, B., y NEVILLE, H. (2009): "Differences in the neural mechanisms of selective attention in children from different socioeconomic backgrounds: An event-related brain potential study."- Developmental science- N 12(4)págs. 634-646.

TILLY, C. (2000): La desigualdad persistente.-Ediciones Manantial- Buenos Aires.

WILKINSON, R., y PICKETT, K. (2009): Desigualdad: Un análisis de la (in)felicidad colectiva. (L. Vidal, Trad.)- TURNER PUBLICACIONES S.L.- Madrid.

YOSHIKAWA, H., ABER, J. L., y BEARDSLEE, W. R. (2012): “The effects of poverty on the mental, emotional, and behavioral health of children and youth: implications for prevention."- The American Psychologist - N 67(4)- págs.272-284. 